\title{
Levantamento etnobotânico das espécies vegetais em quintais de bairro na cidade de Mossoró, Rio Grande do Norte
}

\section{Ethnobotanical survey of plant species in neighborhood quintals in the city of Mossoró, Rio Grande do Norte}

\author{
Maria de Fatima Barbosa Coelho ${ }^{* 1}$,Caio César Pereira Leal ${ }^{2}$, Fabrícia Nascimento de Oliveira ${ }^{3}$, Narjara Walessa Nogueira ${ }^{4}$ \\ Rômulo Magno Oliveira de Freitas ${ }^{5}$
}

Resumo: Os quintais são conhecidos por diferentes denominações em vários lugares e podem ser definidos como uma área de produção localizada perto da casa, onde são cultivadas espécies agrícolas e florestais, com a criação de pequenos animais domésticos. O presente trabalho teve como objetivo realizar um levantamento etnobotânico das espécies vegetais existentes nos quintais do bairro Alto Sumaré em Mossoró, Rio Grande do Norte. A seleção de quinze informantes foi feita usando a técnica de bola de neve e a coleta dos dados foi efetuada com observação direta e participante, turnê guiada, e entrevistas semiestruturadas. Foram observadas 65 espécies pertencentes a 38 famílias botânicas. A família com maior número de espécies foi Lamiaceae e as espécies mais frequentes foram Carica papaya L. e Mangifera indica L. Alocasia macrorrhizos (L.) G. Don e Carica papaya L. foram as espécies mais abundantes. Dos entrevistados, $80 \%$ declararam utilizar plantas medicinais para tratamentos de doenças e 24 espécies têm uso medicinal. As categorias de uso predominantes foram alimentícia, medicinal e ornamental. Mentha X piperita L. e Lippia alba (Mill.) N. E. Br. ex Britton \& P. Wilson foram as mais citadas como medicinal, Carica papaya e Mangifera indica como frutífera e Dieffenbachia picta Schott como ornamental.

Palavras-chave: Caatinga; Plantas medicinais; Sistema agroflorestal.

\begin{abstract}
Homegardens are known by different denominations in various places and can be defined as a production area located near the house, where agricultural and forestry species are grown, with the creation of small domestic animals. The present work had as objective to carry out an ethnobotanical survey of the vegetal species existing in the homegardens of the Alto Sumaré neighborhood in Mossoró, Rio Grande do Norte. The selection of fifteen informants was done using the snowball technique and the data collection was done with direct observation and participant, guided tour, and semi-structured interviews. It was observed 65 species belonging to 38 botanical families. The family with the highest number of species was Lamiaceae and the most frequent species were Carica papaya L. and Mangifera indica L. Alocasia macrorrhizos (L.) G. Don and Carica papaya $\mathrm{L}$. were the most abundant species. Of those interviewed, $80 \%$ reported using medicinal plants for disease treatments and 24 species have medicinal uses. The predominant categories of use were food, medicinal and ornamental. Mentha $X$ piperita L. and Lippia alba (Mill.) N. E. Br. Ex Britton \& P. Wilson were the most cited as medicinal, Carica papaya and Mangifera indica as fruitful and Dieffenbachia picta Schott as ornamental.
\end{abstract}

Key words: Caatinga; Medicinal plants; Agroforestry system.

\footnotetext{
*Autor para correspondência

Recebido para publicação em 14/11/2016; aprovado em 20/12/2016

${ }^{1}$ Profa. Titular, Doutora, Universidade Federal de Mato Grosso, Cuiabá, Mato Grosso, CEP 78060-900. E-mail: coelhomfstrela@ gmail.com

${ }^{2}$ Mestre, Programa de Pós Graduação em Fitotecnia da UFERSA, Mossoró, Rio Grande do Norte, CEP 57625-900. E-mail: caioleal3@ @otmail.com

${ }_{3}^{3}$ Mestre, Programa de Pós Graduação em Fitotecnia da UFERSA, Mossoró, Rio Grande do Norte, CEP 57625-900. E-mail: fabricia@ ufersa.edu.br

${ }^{4}$ Mestre, Programa de Pós Graduação em Fitotecnia da UFERSA, Mossoró, Rio Grande do Norte, CEP 57625-900. E-mail: narjarawalessa@ yahoo.com.br

${ }^{5}$ Mestre, Programa de Pós Graduação em Fitotecnia da UFERSA, Mossoró, Rio Grande do Norte, CEP 57625-900. E-mail: romulomagno_23@hotmail.com
} 


\section{INTRODUÇÃO}

Os sistemas agrícolas quintais são conhecidos por diferentes denominações em vários lugares e podem ser definidos como uma área de produção localizada perto da casa, onde são cultivadas espécies agrícolas e florestais, com a criação de pequenos animais domésticos (KUMAR; NAIR, 2006). As espécies vegetais e animais são manejados na mesma área, simultaneamente ou em uma sequência temporal.

Os quintais tem importância na segurança alimentar e aumento da renda familiar (CARNEIRO et al., 2013; GARCIA et al., 2015), introdução e domesticação de espécies (GAO et al., 2012), eficiência do uso da terra, conservação de espécies e sustentabilidade (KUMAR; NAIR, 2006; FLORENTINO et al., 2007). Os quintais são também responsáveis pela conservação de plantas medicinais por comunidades rurais e urbanas (BAJPAI et al., 2013; PERNA; LAMANO-FERREIRA, 2014).

Nos últimos anos diversos estudos demonstrando a importância dos quintais agroflorestais foram conduzidos no Brasil (FLORENTINO et al., 2007; AMARAL; GUARIM NETO, 2008; EICHEMBERG; AMOROZO, 2009; CARNIELLO et al., 2010; SIVIERO et al., 2011; SEGALA et al., 2012; VIEIRA et al., 2012; FREITAS et al. 2014; MOREIRA; GUARIM NETO, 2015; COELHO et al., 2016), mas a escassez de informações sobre esses sistemas ainda é bastante acentuada, sobretudo no Nordeste e Norte do Brasil (PERNA; LAMANO-FERREIRA, 2014). No Estado do Rio Grande do Norte foram realizados alguns levantamentos etnobotânicos em quintais com ênfase nas plantas medicinais (FREITAS et al., 2012; PAULINO et al., 2012; FREITAS et al., 2014; FREITAS et al., 2015; SILVA et al., 2015; COELHO et al., 2016; FREITAS et al., 2016) e na conservação de espécies do bioma caatinga (ROQUE; LOIOLA, 2013).

Assim, o desenvolvimento de um estudo sobre quintais em Mossoró, Município com grande expressão da agricultura familiar e com comunidades tradicionais, poderá proporcionar a valorização, o resgate e a preservação dos conhecimentos sobre as formas de uso e manejo da biodiversidade local. Portanto, o presente trabalho teve como objetivo realizar um levantamento etnobotânico das espécies vegetais existentes nos quintais do bairro Sumaré em Mossoró, Rio Grande do Norte.

\section{MATERIAL E MÉTODOS}

A pesquisa foi desenvolvida em Mossoró, município localizado nas coordenadas geográficas $5^{\circ} 11^{\prime} \mathrm{S}$ e $37^{\circ} 20^{\prime} \mathrm{W}$, com $18 \mathrm{~m}$ de altitude, no bairro Sumaré e envolvendo 15 quintais no período de abril a junho de 2012.

Para a seleção dos entrevistados foi aplicada a técnica de amostragem de bola de neve (BAILEY, 2008), ou seja, iniciou-se a entrevista com uma das pessoas responsável por quintal e, no final da mesma, solicitou-se que ela indicasse outra, e assim sucessivamente. Foram usadas as técnicas de observação direta e participante e os principais envolvidos no manejo e manutenção dos quintais foram indagadas sobre o uso e manejo das plantas e as partes utilizadas para cada uso (ALBUQUERQUE et al. 2014).

As entrevistas foram feitas também durante a verificação in loco para cada espécie citada por cada informante, utilizando-se o método turnê guiada que consiste em ir até o campo com o informante visando fundamentar e validar os nomes das plantas citadas nas entrevistas anteriores (ALBUQUERQUE et al., 2014).

Em cada quintal foram feitos levantamentos das espécies vegetais com a coleta de amostras para exsicatas e identificação no Herbário Dárdano Andrade Lima da Universidade Federal Rural do Semi Arido.

Depois, as espécies foram classificadas quanto ao uso de acordo com as definições dos próprios entrevistados, evitando-se utilizar categorias pré-definidas e as informações foram compiladas sob a forma de um banco de dados, utilizando-se matrizes de textos, conforme metodologia proposta por Amorozo e Viertler (2010).

\section{RESULTADOS E DISCUSSÃO}

Das pessoas entrevistadas $73 \%$ perteciam ao sexo feminino, $27 \%$ ao sexo masculino e $87 \%$ afirmaram ser casados e $13 \%$ viúvos. A predominância da mulher nas atividades realizadas nos quintais ocorre porque os homens lidam com atividades externas aos arredores das residências, sejam estas remuneradas ou não. Em outros trabalhos sobre quintais, as mulheres também corresponderam à maior parcela dos entrevistados (AMOROZO, 2002; FLORENTINO et al., 2007; MOURA; ANDRADE, 2007; FREITAS et al., 2012).

A condição de casado poderá estar relacionada ao maior conhecimento sobre as plantas, sobretudo as medicinais, pois geralmente a existência dos filhos implica na busca de soluções práticas e imediatas para o tratamento de doenças (FREITAS et al., 2012).

Observou-se que $33 \%$ dos responsáveis pelos quintais encontram-se na faixa etária entre $31-43$ anos de idade (Figura 1A). A pessoa mais nova apresentava 18 anos de idade e a mais velha 79 anos. Em estudo conduzido em Caruaru-PE por Florentino et al. (2007), em Mirassol do Oeste-MT, por Carniello et al. (2010) e em São Miguel-RN, por Freitas et al. (2012) foi observada faixa etária semelhante.

Figura 1. Idade (A) e tempo que os moradores residem (B) no Bairro Alto Sumaré, em Mossoró, Rio Grande do Norte
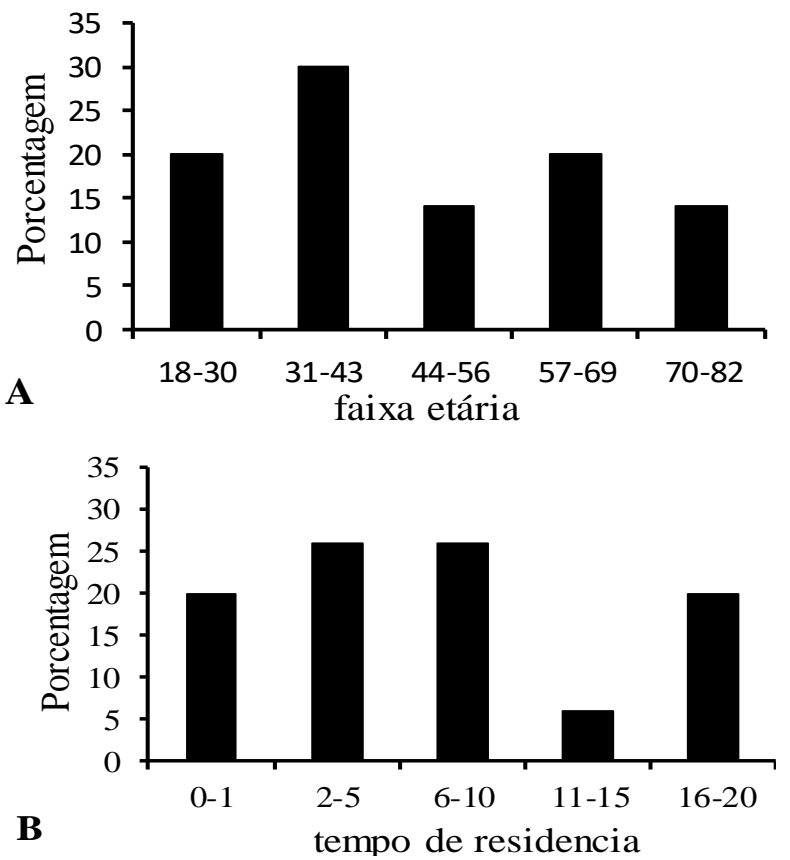
O bairro onde foi realizada a pesquisa encontra-se em construção e apresenta moradores antigos e novos, $20 \%$ dos moradores estão no bairro a menos de 1 ano e o período de até 10 anos concentra 53\% dos moradores (Figura 1B). Dos entrevistados $67 \%$ afirmaram ser naturais da mesma cidade em que residem, as demais cidades, Upanema-RN, Apodi$\mathrm{RN}$, Conceição-BA, Itapipoca-CE e Fortaleza-CE cada uma com $6,6 \%$ dos entrevistados. O local de nascimento e crescimento das pessoas pode implicar diretamente no seu conhecimento acerca dos recursos vegetais e animais de determinada região, de vez que o sistema de classificação, o uso, o manejo, a nomenclatura e outros fatores variam de acordo com a cultura e o ambiente do local de origem e do atual (FREITAS et al., 2015). Nessas residências o número de moradores por casa variou entre 2 e 5 pessoas, sendo mais frequente quatro pessoas $(33 \%)$, também foram encontradas casas com $2(26,6 \%), 3(26,6 \%)$, e $5(13 \%)$ pessoas no núcleo familiar.

Os moradores dedicam-se a diferentes atividades e afirmaram ser aposentados (26,6\%), doméstica $(33,3 \%)$, cobrador $(6,6 \%)$, empreendedor $(13,3 \%)$, ASG $(6,6)$ e soldado (13,3\%). No Rio Grande do Norte, Morais (2011) relatou semelhante composição da renda familiar. Visbiski (2003) em Imaruí-SC, mostrou que a participação da aposentadoria como principal fonte geradora de renda para $27 \%$ das pessoas.

O grau de escolaridade dos informantes encontra-se na Figura 2. Verifica-se que que o mais representativo foram os que afirmaram apresentar o ensino médio e fundamental incompletos, com $27 \%$ das pessoas. Observa-se que $7 \%$ das pessoas afirmaram ter o ensino superior, como também, este mesmo percentual são pessoas analfabetas e dessas apenas $7 \%$ exerceram atividade agricola. A baixa escolaridade também foi encontrada em outros estudos (AMARAL; GUARIM NETO, 2008; CARNIELLO et al., 2010; FREITAS et al., 2012; FREITAS et al., 2015).

Figura 2. Grau de escolaridade de entrevistados no Bairro Alto Sumaré, em Mossoró, Rio Grande do Norte.

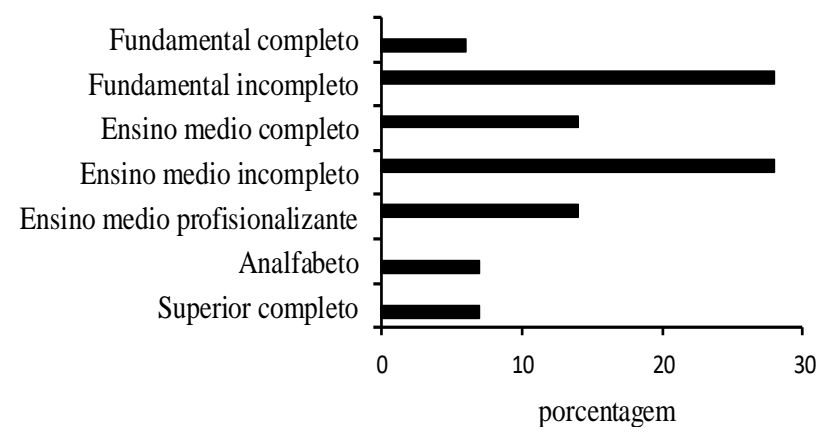

Quando questionados sobre quais espaços compõem a residência, 50\% usaram o termo quintais (Figura 3). Também foram citados jardim (16\%), terreiro (11\%) e muro (22\%). Os arredores das residências estudadas são marcados pela presença de diferentes espaços, percebidos, definidos e utilizados de acordo com fatores socioeconômicos e culturais, tanto dos proprietários e sua família, como aqueles presentes na população local. Freitas (2012) verificou a presença de 15 diferentes espaços reconhecidos e manejados pelos entrevistados, e entre eles também estavam presentes as categorias encontradas no presente estudo.
Figura 3. Nomes dos espaços existentes na residência (b) de entrevistados no Bairro Sumaré, em Mossoró, Rio Grande do Norte

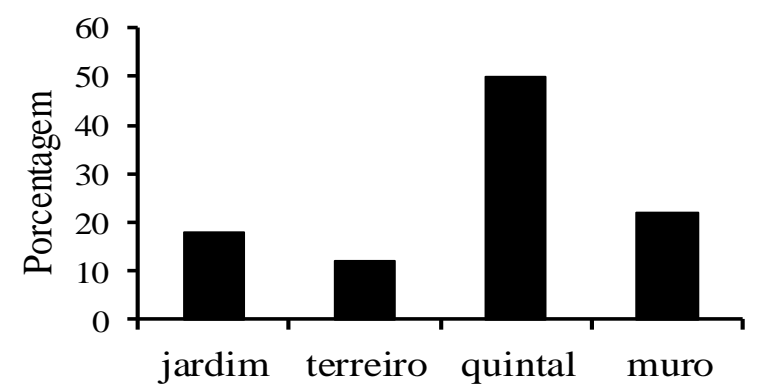

São utilizadas pelos responsáveis pelos quintais técnicas simples de manejo, como capinas periódicas, irrigação manual, limpeza e adubação. Desses, $40 \%$ afirmaram utilizar algum insumo agrícola nessas plantas, sendo o esterco bovino o insumo mais utilizado (100\%). Essas práticas de manejo são encontradas em vários estudos sobre quintais (AMARAL; GUARIM NETO, 2008; CARNIELLO et al., 2010; FREITAS et al., 2016).

De acordo com os entrevistados os materiais vegetais foram obtidos de familiares $(28 \%)$, amigos (24\%), vizinhos $(19 \%)$, feiras $(9,5 \%)$, prefeitura $(4,7 \%)$ e UFERSA $(14,2 \%)$. Familiares e vizinhos são os principais fornecedores de mudas e sementes como também foi verificado por Freitas et al., (2015), Silva (2015); Coelho et al., (2016).

O tamanho dos lotes varia de $10 \times 20 \mathrm{~m}\left(200 \mathrm{~m}^{2}\right)$ a $15 \times 30 \mathrm{~m}\left(450 \mathrm{~m}^{2}\right)$. Áreas com essa extensão são frequentes em loteamentos urbanos no Brasil, e o tamanho não está relacionado com a diversidade e abundancia de espécies, pois se encontram quintais pequenos com grande numero de espécies e quintais grandes com poucas espécies (FREITAS et al., 2012).

Foram encontradas 70 etnoespécies e 65 táxons pertencentes a 38 famílias botânicas (Tabela 1). A família com maior numero de espécies foi Lamiaceae (8 espécies) seguida da Euphorbiaceae (5 espécies). A maior representatividade da família Lamiaceae pode ser explicada por sua distribuição cosmopolita e sua grande importância terapêutica, comprovada por Mosca e Loiola, (2009), Freitas et al. (2012), Paulino et al. (2012), Alves e Povh, (2013), Moreira e Guarim Neto (2015). As espécies desta família se destacam por serem ricas em óleos essenciais, o que confere a estas espécies propriedades aromáticas e medicinais (ABDEL-MOGIBET et al., 2002).

As espécies mais frequentes foram Carica papaya L. e Mangifera indica L., presentes em 8 e 7 quintais respectivamente, já o Cocus nucifera L, Mentha X piperita L., Lippia alba (Mill.) N. E. Br. ex Britton \& P. Wilson e Citrus $X$ limonia (L.) Osbeck foram encontrados em 6 quintais. Alocasia macrorrhizos (L.) G. Don e Carica papaya L. foram as espécies que mais se destacaram em quantidade de plantas, verificando-se, 61 e 25 indivíduos, respectivamente. Em seguida, destacaram-se Musa paradisiaca L., Catharanthus roseus (L.) G. Don. e Cereus peruvianus L. com 20 indivíduos e indicações de uso alimentício e ornamental, respectivamente. 
Tabela 1. Etnoespécies presentes nos quintais do Bairro Sumaré, em Mossoró, Rio Grande do Norte

\begin{tabular}{|c|c|c|c|c|c|}
\hline Família/Espécie & Etnoespécie & $\begin{array}{c}\mathrm{N}^{\circ} \text { de } \\
\text { quintais }\end{array}$ & $\begin{array}{c}\mathrm{N}^{\mathbf{0}} \\
\text { indivíduos }\end{array}$ & $\begin{array}{c}\text { Hábito de } \\
\text { crescimento }\end{array}$ & Uso \\
\hline \multicolumn{6}{|l|}{ Amaranthaceae } \\
\hline Chenopodium ambrosioides L. & Mastruz & 3 & 4 & herbáceo & $\mathrm{b}$ \\
\hline Alternanthera tenella Colla & Cabeça de velho & 1 & 1 & herbáceo & $\mathrm{c}$ \\
\hline \multicolumn{6}{|l|}{ Amaryllidaceae } \\
\hline $\begin{array}{l}\text { Crinum erubescens } \mathrm{L} . \\
\text { Anacardiaceae }\end{array}$ & \multicolumn{4}{|c|}{ Anacardiaceae } & $\mathrm{c}$ \\
\hline Anacardium occidentale L. & Cajueiro & 4 & 4 & arbóreo & $a, b$ \\
\hline Mangifera indica $\mathrm{L}$. & Manga & 7 & 10 & arbóreo & $\mathrm{a}$ \\
\hline Spondias purpurea $\mathrm{L}$. & Seriguela & 3 & 3 & arbóreo & $\mathrm{a}$ \\
\hline $\begin{array}{l}\text { Spondias tuberosa Arr. Cam. } \\
\text { Annonaceae }\end{array}$ & \multicolumn{4}{|c|}{ Annonaceae } & a \\
\hline Annona squamosa L. & Pinha & 1 & 1 & arbustivo & a \\
\hline $\begin{array}{l}\text { Annona muricata } \mathrm{L} \text {. } \\
\text { Apocynaceae }\end{array}$ & Graviola & 4 & 6 & arbustivo & $\mathrm{a}, \mathrm{b}$ \\
\hline Catharanthus roseus (L.) G. Don. & Boa noite & 1 & 20 & herbáceo & $\mathrm{c}$ \\
\hline $\begin{array}{l}\text { Catharanthus roseus (L.) G. Don. } \\
\text { Araceae }\end{array}$ & Onze horas & 1 & 1 & herbáceo & $\mathrm{c}$ \\
\hline Alocasia macrorrhizos (L.) G. Don & Croton & 1 & 61 & herbáceo & $\mathrm{c}$ \\
\hline $\begin{array}{l}\text { Dieffenbachia picta Schott } \\
\text { Arecaceae }\end{array}$ & Comigo ninguém pode & 4 & 8 & herbáceo & $\mathrm{c}$ \\
\hline Cocos nucifera $\mathrm{L}$. & Coqueiro anão & 3 & 5 & arbóreo & a \\
\hline Cocos nucifera $\mathrm{L}$. & Coqueiro gigante & 6 & 17 & arbóreo & $\mathrm{a}, \mathrm{c}$ \\
\hline \multicolumn{6}{|l|}{ Asphodelaceae } \\
\hline \multicolumn{6}{|l|}{ Asteraceae } \\
\hline \multicolumn{6}{|l|}{ Balsaminaceae } \\
\hline $\begin{array}{l}\text { Impatiens balsamina } \mathrm{L} \text {. } \\
\text { Bromeliaceae }\end{array}$ & Milingu & 1 & 1 & herbáceo & $\mathrm{c}$ \\
\hline $\begin{array}{l}\text { Ananas comosus (L.) Merr. } \\
\text { Cactaceae }\end{array}$ & \multicolumn{4}{|c|}{ Cactaceae } & $\mathrm{c}$ \\
\hline Opuntia ficus-indica Mill & Palma & 1 & 1 & arbóreo & $\mathrm{c}$ \\
\hline Cereus peruvianus L. & Cardeiro & 1 & 1 & arbóreo & $\mathrm{c}$ \\
\hline $\begin{array}{l}\text { Cereus peruvianus L. } \\
\text { Caricaceae }\end{array}$ & Cacto & 2 & 20 & arbóreo & $\mathrm{c}$ \\
\hline $\begin{array}{l}\text { Carica papaya } \mathrm{L} . \\
\text { Crassulaceae }\end{array}$ & Mamão & 8 & 25 & arbóreo & $\mathrm{a}, \mathrm{b}$ \\
\hline \multicolumn{5}{|l|}{ Cucurbitaceae } & $\mathrm{b}$ \\
\hline \multicolumn{6}{|l|}{ Euphorbiaceae } \\
\hline Jathropha curcas L. & Pinhão bravo & 1 & 2 & arbustivo & $\mathrm{c}$ \\
\hline Jathropha curcas L. & Pinhão manso & 1 & 1 & arbustivo & $\mathrm{c}$ \\
\hline Jatropha gossypiifolia L. & Pinhão do Pará & 1 & 1 & arbustivo & $\mathrm{c}$ \\
\hline Manihot utilissima Pohl & Macaxeira & 1 & 4 & arbustivo & $\mathrm{a}$ \\
\hline \multicolumn{5}{|l|}{ Lamiaceae } & $\mathrm{c}$ \\
\hline Rosmarinus officinalis L. & Alecrim & 1 & 1 & herbáceo & $\mathrm{b}$ \\
\hline Justicia pectoralis L. & Anador & 1 & 5 & herbáceo & $\mathrm{b}$ \\
\hline Ruta graveolens L. & Arruda & 1 & 1 & herbáceo & $\mathrm{b}$ \\
\hline Plectranthus barbatus Andrews & Boldo & 3 & 3 & herbáceo & $\mathrm{b}$ \\
\hline Ocimum gratissimum $\mathrm{L}$. & Louro & 1 & 5 & herbáceo & $\mathrm{b}$ \\
\hline Plectranthus amboinicus (Lour.) Spreng. & Malva & 5 & 6 & herbáceo & $\mathrm{b}$ \\
\hline Mentha X piperita $\mathrm{L}$. & Hortelã & 6 & 9 & herbáceo & $a, b$ \\
\hline $\begin{array}{l}\text { Ocimum basilicum } \mathrm{L} . \\
\text { Lauraceae }\end{array}$ & Manjericão & 2 & 3 & herbáceo & $\mathrm{b}$ \\
\hline $\begin{array}{l}\text { Persea americana Mill. } \\
\text { Leguminosae - Papilionoideae }\end{array}$ & Abacateiro & 2 & 2 & arbóreo & $\mathrm{a}$ \\
\hline Erytrina indica picta L. & Brasileirinho & 1 & 1 & arbóreo & $\mathrm{c}$ \\
\hline
\end{tabular}




\begin{tabular}{|c|c|c|c|c|c|}
\hline $\begin{array}{l}\text { Lythraceae } \\
\text { Punica granatum L. } \\
\text { Malpighiaceae }\end{array}$ & Romã & 4 & 4 & arbustivo & $\mathrm{b}$ \\
\hline $\begin{array}{l}\text { Malpighia glabra L. } \\
\text { Meliaceae }\end{array}$ & Acerola & 4 & 9 & arbustivo & $\mathrm{a}$ \\
\hline $\begin{array}{l}\text { Azadirachta indica A. Juss } \\
\text { Moraceae }\end{array}$ & $\mathrm{Nim}$ & 3 & 7 & arbóreo & $\mathrm{c}$ \\
\hline Ficus benjamina L. & Sempre verde & 1 & 1 & arbóreo & $\mathrm{c}$ \\
\hline $\begin{array}{l}\text { Rubus spp. } \\
\text { Musaceae }\end{array}$ & Amora miúda & 1 & 1 & arbustivo & $\mathrm{b}$ \\
\hline $\begin{array}{l}\text { Musa paradisiaca } \mathrm{L} \text {. } \\
\text { Myrtaceae }\end{array}$ & Bananeira & 4 & 20 & arbóreo & $\mathrm{a}$ \\
\hline Syzygium cumini (L.) Skeels & Azeitona roxa & 1 & 1 & arbóreo & $\mathrm{a}$ \\
\hline $\begin{array}{l}\text { Psidium guajava } \mathrm{L} . \\
\text { Oleaceae }\end{array}$ & Goiabeira & 5 & 8 & arbóreo & $\mathrm{a}, \mathrm{b}$ \\
\hline $\begin{array}{l}\text { Jasminum sambac (L.) Aiton } \\
\text { Oxalidaceae }\end{array}$ & Bugari & 1 & 1 & arbóreo & $\mathrm{c}$ \\
\hline $\begin{array}{l}\text { Averrhoa carambola } \mathrm{L} . \\
\text { Oxalis tetraphylla } \mathrm{L} .\end{array}$ & $\begin{array}{l}\text { Carambola } \\
\text { Trevo }\end{array}$ & $\begin{array}{l}1 \\
1\end{array}$ & $\begin{array}{l}2 \\
1\end{array}$ & $\begin{array}{l}\text { arbóreo } \\
\text { herbáceo }\end{array}$ & $\begin{array}{l}\mathrm{a} \\
\mathrm{c}\end{array}$ \\
\hline Phyllanthaceae & & & & & \\
\hline $\begin{array}{l}\text { Phyllanthus niruri } \mathrm{L} . \\
\text { Phyllanthus niruri } \mathrm{L} . \\
\text { Poaceae }\end{array}$ & $\begin{array}{l}\text { Quebra-pedra } \\
\text { Quebra-pedra do Pará }\end{array}$ & $\begin{array}{l}2 \\
1\end{array}$ & $\begin{array}{l}2 \\
1\end{array}$ & $\begin{array}{l}\text { herbáceo } \\
\text { herbáceo }\end{array}$ & $\begin{array}{l}\mathrm{b} \\
\mathrm{b}\end{array}$ \\
\hline $\begin{array}{l}\text { Cymbopogon citratus (DC.) Stapf } \\
\text { Polypodiaceae }\end{array}$ & Capim santo & 5 & 8 & herbáceo & $\mathrm{b}$ \\
\hline $\begin{array}{l}\text { Pilea microphyla L. } \\
\text { Polypodium lepidopteris (Langds. \& Fisch.) } \\
\text { Kunze } \\
\text { Rosaceae }\end{array}$ & $\begin{array}{l}\text { Planta de sorte } \\
\text { Samambaia }\end{array}$ & $\begin{array}{l}1 \\
2\end{array}$ & $\begin{array}{l}3 \\
8\end{array}$ & $\begin{array}{l}\text { herbáceo } \\
\text { herbáceo }\end{array}$ & $\begin{array}{l}\mathrm{c} \\
\mathrm{c}\end{array}$ \\
\hline $\begin{array}{l}\text { Rosa chinensis Jacq. } \\
\text { Rosa chinensis Jacq. } \\
\text { Rubiaceae }\end{array}$ & $\begin{array}{l}\text { Rosa bendita } \\
\text { Roseira }\end{array}$ & $\begin{array}{l}1 \\
2\end{array}$ & $\begin{array}{l}5 \\
2\end{array}$ & $\begin{array}{l}\text { arbustivo } \\
\text { arbustivo }\end{array}$ & $\begin{array}{l}\mathrm{c} \\
\mathrm{c}\end{array}$ \\
\hline $\begin{array}{l}\text { Ixora coccinea } \mathrm{L} . \\
\text { Morinda citrifolia } \mathrm{L} . \\
\text { Ruscaceae }\end{array}$ & $\begin{array}{l}\text { Ixore } \\
\text { Noni }\end{array}$ & $\begin{array}{l}1 \\
3\end{array}$ & $\begin{array}{l}4 \\
6\end{array}$ & $\begin{array}{l}\text { arbustivo } \\
\text { arbóreo }\end{array}$ & $\begin{array}{c}c \\
a, b\end{array}$ \\
\hline $\begin{array}{l}\text { Sansevieria trifasciata Prain 'Hahnii' } \\
\text { Rutaceae }\end{array}$ & Espada de São Jorge & 3 & 6 & herbáceo & $\mathrm{c}$ \\
\hline $\begin{array}{l}\text { Citrus sinensis (L.) Osbeck } \\
\text { Citrus X limonia (L.) Osbeck } \\
\text { Solanaceae }\end{array}$ & $\begin{array}{l}\text { Laranjeira } \\
\text { Limoeiro }\end{array}$ & $\begin{array}{l}2 \\
6\end{array}$ & $\begin{array}{l}2 \\
9\end{array}$ & $\begin{array}{l}\text { arbóreo } \\
\text { arbóreo }\end{array}$ & $\begin{array}{c}a \\
a, b\end{array}$ \\
\hline $\begin{array}{l}\text { Capsicum chinense Jacq. } \\
\text { Capsicum frutescens } \mathrm{L} \text {. } \\
\text { Urticaceae }\end{array}$ & $\begin{array}{l}\text { Pimenta de cheiro } \\
\text { Pimenta malagueta }\end{array}$ & $\begin{array}{l}1 \\
1\end{array}$ & $\begin{array}{l}1 \\
1\end{array}$ & $\begin{array}{l}\text { herbáceo } \\
\text { herbáceo }\end{array}$ & $\begin{array}{l}\mathrm{a} \\
\mathrm{d}\end{array}$ \\
\hline $\begin{array}{l}\text { Pilea microphylla (L.) Liebm. } \\
\text { Verbenaceae }\end{array}$ & Orelha de rato & 1 & 1 & herbáceo & $\mathrm{c}$ \\
\hline $\begin{array}{l}\text { Lippia alba (Mill.) N. E. Br. ex Britton \& P. } \\
\text { Wilson }\end{array}$ & Erva cidreira & 6 & 9 & arbustivo & $\mathrm{b}$ \\
\hline $\begin{array}{l}\text { Duranta repens } \mathrm{L} \text {. } \\
\text { Vitaceae }\end{array}$ & Pingo de ouro & 1 & 1 & arbustivo & $\mathrm{c}$ \\
\hline Vitis vinifera L. & Uva & 1 & 1 & trepadeira & $\mathrm{a}$ \\
\hline Total & & & 387 & & \\
\hline
\end{tabular}

Considerando todos os quintais estudados no Bairro Alto Sumaré, registrou-se a presença de 387 plantas, com média de 25 plantas por quintal. Verifica-se que esse número de plantas é pequeno quando comparado com o estudo de Freitas et al., (2016) que encontraram em 20 quintais 1.447 indivíduos, com uma média de 72 plantas por quintal e Florentino et al., (2007) que em 25 quintais de Caruaru-PE encontraram 1.461 indivíduos. Esta diferença deve-se possivelmente à proximidade que estes quintais encontram-se da zona agrícola, facilitando a obtenção de diferentes espécies, e à maior extensão dos lotes nestes trabalhos.
A predominância no hábito herbáceo foi verificada nos quintais e pode estar associada com o fácil cultivo das ervas, facilitando a obtenção desses recursos vegetais pelos moradores (PILLA et al., 2006; ALBERTASSE et al., 2010).

Nesses espaços foram encontradas várias categorias de uso das plantas (Figura 4), sendo que 33\% apresentaram medicinais, $31 \%$ frutíferas e $25 \%$ ornamentais $(25 \%)$. Estas categorias são frequentemente citadas como as mais importantes em outros estudos realizados no Rio Grande do Norte (FREITAS et al., 2012; PAULINO et al., 2012; 
FREITAS et al., 2014; FREITAS et al., 2015; SILVA et al., 2015; COELHO et al., 2016; FREITAS et al., 2016).

O uso de espécies como medicinal é importante, pois entre os responsáveis pelos quintais $80 \%$ declararam utilizar plantas para tratamento de doenças. Segundo os mesmos o conhecimento sobre essas plantas foi obtido através de familiares $(40 \%)$, pela tradição $(26,6 \%)$, pelas pessoas mais idosas $(13,3 \%)$ e através da própria prática $(13,3 \%)$.

Figura 4. Categorias de plantas e porcentagem dos quintais do Bairro Alto Sumaré, em Mossoró, Rio Grande do Norte

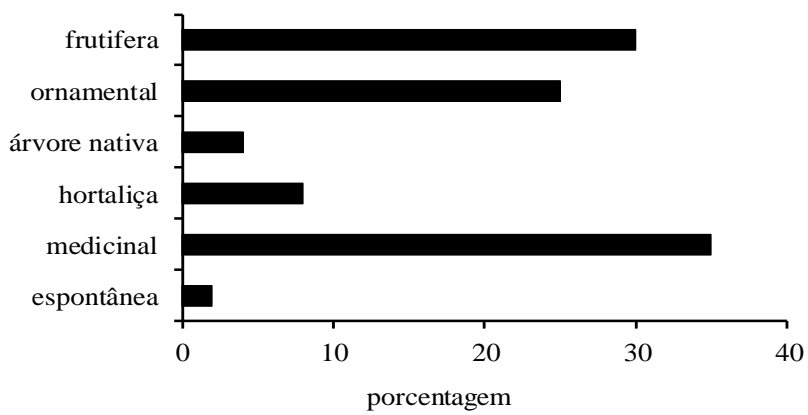

Das pessoas entrevistadas no bairro, $61 \%$ afirmaram que quando precisam de médicos procuram o posto de saúde do bairro e os demais disseram utilizar clínicas particulares $(27,7 \%)$ e hospitais da rede pública. Dessas pessoas, $67 \%$ afirmaram não utilizar medicamentos convencionais e plantas medicinais de forma simultânea, no entanto, se o médico recomendar à utilização de remédios à base de plantas medicinais, $73 \%$ dos entrevistados seguiria o tratamento.

Quando questionados sobre o que consideram mais confiáveis, um tratamento a base de plantas medicinais ou um com medicamentos convencionais, 53\% dos entrevistados responderam que o tratamento convencional é mais confiável. Porém, quando se levantou a situação de haver duas farmácias, uma com medicamentos convencionais e outra com medicamentos a base de plantas medicinais, 53\% preferiam o medicamento a base de plantas medicinais.

A alta frequência de plantas utilizadas na alimentação concorda com os resultados encontrados na literatura (FLORENTINO et al., 2007; SILVA et al., 2014; GARCIA et al., 2015; COELHO et al., 2016). Os quintais exercem um papel importante para a segurança alimentar dos agricultores, pois a riqueza de espécies encontradas nos extratos arbóreos e arbustivos, representados em sua maioria por espécies alimentícias que proporcionam alimentação saudável, com riqueza de nutrientes (GARCIA et al., 2015). As verduras, frutas e legumes constituem valiosas fontes de nutrientes para a família, uma alternativa econômica para o consumo de produtos em épocas de crise, e que, de outra maneira, seria de difícil obtenção (VALADÃO et al. 2006).

A frequência absoluta e relativa dos usos das etnoespécies por quintais encontram-se na Tabela 2. De acordo com esses resultados, observa-se a predominância das plantas alimentícias e medicinais com respectivamente $42,8 \%$ e $42,1 \%$ de plantas.

Esses resultados corroboram com os encontrados por outros autores que constataram que as espécies mais frequentes nos quintais são as alimentícias e medicinais (FLORENTINO et al., 2007; AMARAL; GUARIM NETO, 2008; CARNIELLO et al. 2010; PASA, 2011; FREITAS et al 2012). Um dos estudos que chamam a atenção para as espécies alimentícias foi conduzido em Rosário Oeste-MT por Amaral e Guarim Neto (2008) que identificaram 94 espécies vegetais cultivadas com a finalidade de complementação da dieta alimentar representadas por espécies frutíferas, como o cajueiro (Anacardium ocidentalle), o mamoeiro (Carica papaya) e a bananeira (Musa paradisiaca).

Pode-se verificar também que os quintais diferem com relação à composição das plantas, como por exemplo, o quintal 3 com 23 etnoespécies das quais 8 são alimentícias e 14 são medicinais, enquanto o quintal 6 apresenta a menor diversidade com 5 etnoespécies das quais 2 são alimentícias e 3 são medicinais.

Os dados referentes às espécies citadas como medicinais no Bairro Alto Sumaré encontram-se na Tabela 3. Foram citadas 24 etnoespécies com propriedades medicinais, representando $42,1 \%$ do total de etnoespécies encontradas, e destas, as mais citadas nas entrevistas foram Mentha $X$ piperita e Lippia alba, por $40 \%$ dos entrevistados. Ressalta-se que a Mentha $X$ piperita, Lippia alba e Carica papaya destacaram-se como as etnoespécies mais abundantes, com um número total de 9 plantas, correspondendo a $8,6 \%$ do total.

Tabela 2 - Frequência das etnoespécies por quintal e categoria de uso no Bairro Sumaré, em Mossoró, Rio Grande do Norte

\begin{tabular}{|c|c|c|c|c|c|c|c|c|c|c|}
\hline \multirow{2}{*}{ Quintal } & \multirow{2}{*}{$\mathrm{N}^{\mathrm{o}}$ total de etnoespécies citadas } & \multicolumn{8}{|c|}{$\mathrm{N}^{\circ}$ de etnoespécies citadas por categorias de uso } & \multirow{2}{*}{ Total de usos } \\
\hline & & Alim. & $\%$ & Med. & $\%$ & Orn. & $\%$ & Out. & $\%$ & \\
\hline Q1 & 14 & 04 & 28,6 & 0 & 0 & 10 & 71,4 & 0 & 0 & 2 \\
\hline Q2 & 07 & 03 & 42,9 & 01 & 14,3 & 02 & 28,6 & 1 & 14,3 & 4 \\
\hline Q3 & 23 & 08 & 34,8 & 14 & 60,9 & 03 & 13,0 & 1 & 4,3 & 4 \\
\hline Q4 & 09 & 0 & 0 & 03 & 33,3 & 06 & 66,7 & 0 & 0 & 2 \\
\hline Q5 & 06 & 0 & 0 & 0 & 0 & 06 & 100 & 0 & 0 & 1 \\
\hline Q6 & 05 & 02 & 40 & 03 & 60 & 0 & 0 & 0 & 0 & 2 \\
\hline Q7 & 17 & 09 & 52,9 & 05 & 29,5 & 03 & 17,6 & 0 & 0 & 3 \\
\hline Q8 & 07 & 06 & 85,7 & 04 & 57,1 & 0 & 0 & 0 & 0 & 2 \\
\hline Q9 & 14 & 09 & 64,3 & 07 & 50,0 & 02 & 14,3 & 0 & 0 & 3 \\
\hline Q10 & 08 & 06 & 75,0 & 02 & 25,0 & 01 & 12,5 & 0 & 0 & 3 \\
\hline Q11 & 11 & 08 & 72,7 & 04 & 36,4 & 01 & 9,0 & 0 & 0 & 3 \\
\hline Q12 & 06 & 02 & 33,3 & 0 & 0 & 04 & 66,7 & 0 & 0 & 2 \\
\hline Q13 & 12 & 07 & 58,3 & 05 & 41,7 & 01 & 8,3 & 01 & 8,3 & 4 \\
\hline Q14 & 19 & 01 & 5,3 & 11 & 57,9 & 07 & 36,8 & 0 & 0 & 3 \\
\hline Q15 & 11 & 03 & 27,3 & 08 & 72,7 & 0 & 0 & 0 & 0 & 2 \\
\hline TOTAL & 159 & 68 & 42,8 & 67 & 42,1 & 46 & 28,9 & 3 & 1,9 & ,7 \\
\hline
\end{tabular}

Ali= alimentício, Med.=medicinal, Orn. $=$ ornamental, Out. $=$ outros usos 
Tabela 3. Espécies utilizadas para fins terapêuticos pelos moradores do Bairro Sumaré, em Mossoró, Rio Grande do Norte

\begin{tabular}{|c|c|c|c|c|c|}
\hline Espécies & Indicações Terapêuticas Citadas & $\begin{array}{l}\text { Parte } \\
\text { Usada }\end{array}$ & Modo de Preparo & NC & $\mathbf{Q P}$ \\
\hline Aloe vera & Cicatrização de feridas, queimaduras & Folha & Sumo da folha & 5 & 8 \\
\hline $\begin{array}{l}\text { Alternanthera } \\
\text { brasiliana }\end{array}$ & Ferimentos & Folha & In natura & 1 & 1 \\
\hline $\begin{array}{l}\text { Anacardium } \\
\text { occidentale }\end{array}$ & Ferida no útero, ferimentos externos, inflamação & Casca & Lambedor & 2 & 2 \\
\hline Annona muricata & Câncer & Folha & Chá & 1 & 1 \\
\hline Carica papaya & Febre e má digestão & Folha & Chá & 1 & 9 \\
\hline $\begin{array}{l}\text { Chenopodium } \\
\text { ambrosioides }\end{array}$ & Gripe & Folha & Lambedor & 3 & 4 \\
\hline Citrus X limonia & Febre, gripe & Fruto & Suco, Chá & 2 & 2 \\
\hline Cymbopogon citratus & Nervosismo, calmante, insônia, febre & Folha & Chá & 5 & 8 \\
\hline Justicia pectoralis & Dores & Folha & Lambedor & 1 & 5 \\
\hline Kalanchoe brasiliensis & Gripe, Inflamação, ferimentos & Folha & Lambedor, compressa & 4 & 5 \\
\hline Lippia alba & Dor de barriga, calmante & Folha & Chá & 6 & 9 \\
\hline Mentha X piperita & Dor de cabeça, gripe, má digestão, cólica, dor de garganta & Folha & Chá, Lambedor & 6 & 9 \\
\hline Morinda citrifolia & Diabete, gastrite, próstata e câncer & Fruto & Suco, Lambedor & 2 & 4 \\
\hline Ocimum basilicum & Dor de ouvido & Folha & In natura & 2 & 3 \\
\hline Ocimum gratissimum & Má digestão & Folha & Chá & 1 & 5 \\
\hline Phyllanthus niruri & Pedra nos rins & Folha & Chá & 2 & 2 \\
\hline Phyllanthus niruri & Infecção urinária, diurético & Folha & Chá & 1 & 1 \\
\hline $\begin{array}{l}\text { Plectranthus } \\
\text { amboinicus }\end{array}$ & Gripe & Folha & Lambedor, Xarope & 5 & 6 \\
\hline Plectranthus barbatus & Dores estomacais, anti-inflamatório, gripe e anticatarral & Folha & Chá, lambedor & 3 & 3 \\
\hline Psidium guajava & Diarréia & $\begin{array}{l}\text { Folha } \\
\text { nova }\end{array}$ & Chá & 4 & 7 \\
\hline Punica granatum & Dor de garganta & Fruto & Gargarejos, Lambedor & 4 & 4 \\
\hline Rosmarinusofficinalis & Relaxante & $\begin{array}{l}\text { Planta } \\
\text { inteira }\end{array}$ & Chá & 1 & 1 \\
\hline Rubus spp. & Emagrecer & Folha & Chá & 1 & 1 \\
\hline Rutagraveolens & Dor de cólica & Folha & Chá & 1 & 1 \\
\hline
\end{tabular}

$\mathrm{NC}=$ Número de citações; $\mathrm{QP}=$ Quantidade de plantas nos núcleos familiares que consideraram a espécie como medicinal.

Quanto à indicação das plantas medicinais para o tratamento de doenças nos quintais avaliados, os entrevistados citaram um total de 29 doenças/sintomas que podem ser tratadas com espécies medicinais. As indicações terapêuticas mais frequentes foram gripe com 6 citações, seguidas de ferimentos, 4 citações, e febre e má digestão, 3 citações, correspondendo a $20,7 \%, 13,8 \%$ e $10,3 \%$, respectivamente.

As folhas são a parte mais utilizada e o preparo mais frequente foi o chá. A maioria dos autores que trabalha com etnobotânica de plantas medicinais também destaca a folha como a parte vegetal mais utilizada nessas preparações (MOSCA e LOIOLA, 2009; ALBERTASSE et al., 2010; GUERRA et al., 2010; FREITAS et al., 2012). O uso frequente de plantas medicinais herbáceas cultivadas em espaços domésticos pelas pessoas da comunidade pode explicar o uso predominante de folhas. A maioria das espécies vegetais tende a concentrar os princípios ativos nas folhas (STEPP, 2001), o que corrobora a importância do uso destas partes vegetais de plantas medicinais.

\section{CONCLUSÕES}

Neste estudo foram observadas 65 espécies pertencentes a 38 famílias botânicas.

A família com maior número de espécies foi Lamiaceae e as espécies mais frequentes foram Carica papaya e Mangifera indica.
Alocasia macrorrhizos e Carica papaya foram as espécies mais abundantes.

Os usos principais das espécies foram alimentício, medicinal e ornamental.

As espécies Mentha $X$ piperita e Lippia alba foram as mais citadas como medicinal, Carica papaya e Mangifera indica como frutífera e Dieffenbachia picta como ornamental.

\section{REFERÊNCIAS}

ABDEL-MOGIB, M.; ALBAR, H. A.; BATTERJEE, S. M. Chemistry of the Genus Plectranthus. Molecules, v.7, p. 271301, 2002.

ALBERTASSE, P. D.; THOMAZ, L. D.; ANDRADE, M. A. Plantas medicinais e seus usos na comunidade da Barra do Jucu, Vila Velha, ES. Revista Brasileira de Plantas Medicinais, Campinas, v.12, n.3, p.250-260, 2010.

ALBUQUERQUE, U. P.; CUNHA, L. V. F. C.; LUCENA, R. F. P.; ALVES, R. R. N. (Eds.) Methods and Techniques in Ethnobiology and Ethnoecology, Springer Protocols Handbooks, 2014, 480 p.

ALVES, G. S. P.; POVH, J. A. Estudo etnobotânico de plantas medicinais na comunidade de Santa Rita, Ituiutaba MG. Biotemas, Florianópolis, v.26, n.3, p.231-242, 2013. 
AMARAL, C. N.; GUARIM-NETO, G. Os quintais como espaços de conservação e cultivo de alimentos: um estudo na cidade de Rosário Oeste (Mato Grosso, Brasil). Boletim do Museu Paraense Emílio Goeldi, Belém, v. 3, n.3, p.329-341, 2008.

AMOROZO, M. C. M. Uso e diversidade de plantas medicinais em Santo Antônio do Leverger, MT, Brasil. Acta Botânica Brasílica, Belo Horizonte, v.16, n.2, p.189-203, 2002.

AMOROZO, M. C. M.; VIERTLER, R. B. A abordagem qualitativa na coleta e análise de dados em etnobiologia e etnoecologia. In: Albuquerque, U. P.; Lucena, R. F. P.; Cunha, L. V. F. C. Métodos e técnicas na pesquisa etnobiológica e etnoecológica. Recife: NUPEEA, 2010. p. $67-$ 82 .

BAILEY, K. Methods of Social Research. 4. ed. New York: The Free Press, 2008. 592p.

BAJPAI, S.; SHARMA, A. K.; KANUNGO, V. K. Traditional home gardens: A preserve of medicinal plants. International Journal of Herbal Medicine, v.1, n.2, p.152161, 2013.

CARNEIRO, M. G. R.; CAMURÇA, A. M.; ESMERALDO, G. G. S. L.; SOUSA, N. R. Quintais Produtivos: contribuição à segurança alimentar e ao desenvolvimento sustentável local na perspectiva da agricultura familiar ( $\mathrm{O}$ caso do Assentamento Alegre, Município de Quixeramobim/CE). Revista Brasileira de Agroecologia, Porto Alegre, v.8, n.2, p.135-147, 2013.

CARNIELlO, M. A.; SILVA, R. S.; CRUZ, M. A. B.; GUARIM NETO, G. Quintais urbanos de Mirassol D’Oeste-MT, Brasil: uma abordagem etnobotânica. Acta Amazônica, Manaus, v.40, n.3, p.451-470, 2010.

COELHO, M. F. B.; FREITAS, A. V. L.; PEREIRA, Y. B.; FREITAS NETO, E. C.; AZEVEDO, R. A. B. Production of food and medicinal plants in homegardens in Northeast of Brazil. International Journal of Current Multidisciplinary Studies, v.2, n.4, p.191-199, 2016.

EICHEMBERG, M. T.; AMOROZO, M. C. M.; MOURA, L. C. Species composition and plant use in old urban homegardens in Rio Claro, Southeast of Brazil. Acta Botânica Brasílica, Belo Horizonte, v.23, n.4, p.:1057-1075, 2009.

FLORENTINO, A. T. N.; ARAUJO, E. L.; ALBUQUERQUE, U. P. Contribuição de quintais agroflorestais na conservação de plantas da Caatinga, Município de Caruaru, PE, Brasil. Acta Botânica Brasílica, Belo Horizonte, v.21, p.37-47, 2007.

FREITAS, A. V. L.; COELHO, M. F. B.; PEREIRA, Y. B.; FREITAS NETO, E. C.; AZEVEDO, R. A. B. Diversidade e uso de plantas medicinais nos quintais da comunidade de São João da Várzea em Mossoró, RN. Revista Brasileira de Plantas Medicinais, v.17, n.4, p.845-856, 2015.

FREITAS, A. V. L.; AZEVEDO, R. A. B.; PEREIRA, Y. B.; FREITAS NETO, E. C.; COELHO, M. F. B. Uses of medicinal plants in Rio Grande do Norte, Brazil. Journal of Global Bioscience, v.3, n.4, p.749-762, 2014.

FREITAS, A. V. L.; COELHO, M. F. B.; MAIA, S. S. S.; AZEVEDO, R. A. B. Plantas medicinais: um estudo etnobotânico nos quintais do Sítio Cruz, São Miguel, Rio Grande do Norte, Brasil. Revista Brasileira de Biociências, Porto Alegre, v.10, n.1, p.48-59, 2012.

FREITAS, A. V. L.; COELHO, M. F. B.; PEREIRA, Y. P.; FREITAS NETO, E. C.; AZEVEDO, R. A. B. Sitio Cruz homegardens in São Miguel, Rio Grande do Norte, Brazil. Journal of Global Biosciences, v.5, n.8, p.4451-4462, 2016.

GAO, J.; HE, T.; LI, Q. M. Traditional home-garden conserving genetic diversity: a case study of Acacia pennata in southwest China. Conservation Genetics, v.13, n.4, p.891$898,2012$.

GUERRA, A. M. N. M.; PESSOA, M. F.; SOUZA, C. S. M.; MARACAJÁ, P. B. Utilização de plantas medicinais pela comunidade rural Moacir Lucena, Apodi-RN. Bioscience Journal,Uberlândia, v.26, n.3, p.442-450, 2010.

KUMAR, B. M.; NAIR, P. K. R. Tropical home gardens: A time tested example of sustainable agroforestry. Springer Science, Dordrecht, The Netherlands, 2006, 380p.

MOSCA, V. P.; LOIOLA, M. I. B. Uso popular de plantas medicinais no Rio Grande do Norte, nordeste do Brasil. Revista Caatinga, Mossoró, v.22, n. , p.225-234, 2009.

MOREIRA, R. P. M.; GUARIM NETO, G. A flora medicinal dos quintais de Tangará da Serra, Mato Grosso, Brasil. Biodiversidade, Rondonópolis, v.14, n.1, p.63-83, 2015.

MOURA, C. L.; ANDRADE, L. H. A. Etnobotânica em quintais urbanos nordestinos: um estudo no bairro da Muribeca, Jaboatão dos Guararapes, PE. Revista Brasileira de Biociências, Porto Alegre, v.5, n.1, p.219-221, 2007.

PASA, M. G. O saber local e medicina popular: a etnobotânica em Cuiabá, Mato Grosso Brasil. Boletim do Museu Paraense Emílio Goeldi. Ciências Humanas, Belem, v.6, n.1, p.179-196, 2011.

PAULINO, R. C.; HENRIQUES, G. P. S. A.; MOURA, O. N. S.; COELHO, M. F. B.; AZEVEDO, R. A. B. Medicinal plants at the Sítio do Gois, Apodi, Rio Grande do Norte State, Brazil. Revista Brasileira de Farmacognosia, Curitiba, v.22, n.1, p.29-39, 2012.

PILlA, M. A. C.; AMOROZO, M. C. M.; FURLAN, A. Obtenção e uso das plantas medicinais no distrito de Martim Francisco, Município de Mogi-Mirim, SP, Brasil. Acta Botânica Brasílica, São Paulo, v.20, n.4, p. 789-802, 2006.

PERNA, T. A.; LAMANO-FERREIRA, A. P. N. Revisão Bibliométrica Sobre o Cultivo de Plantas Medicinais em Quintais Urbanos em Diferentes Regiões do Brasil (20092012). UNOPAR Científica Ciências Biológicas e da Saúde, Londrina, v.16, n.1, p.61-67, 2014.

GARCIA, B. N. R.; VIEIRA, T. A.; OLIVEIRA, F. A. Quintais agroflorestais e segurança alimentar em uma 
comunidade rural na Amazônia Oriental. Rev. Fac. Agron. La Plata. v.114, n.1, p.67-73, 2015.

ROQUE, A. A.; ROCHA, R. M.; LOIOLA, M. I. B. Uso e diversidade de plantas medicinais da Caatinga na comunidade rural de Laginhas, município de Caicó, Rio Grande do Norte (Nordeste do Brasil). Revista Brasileira de Plantas Medicinais, Campinas, v.12, n.1, p.31-42, 2010.

SEGAllA, R.; COELHO, M. F. B; AZEVEDO, R. A. B. Agricultura urbana em Área de Preservação Permanente. Revista Trópica - Ciências Agrárias e Biológicas, São Luis, v.6, n.1, p.17-29, 2012.

SILVA, A. C.; MAIA, S. S. S.; COELHO, M. B. C.; SILVA, R. C. P.; CANDIDO, W. S. Medicinal Plants Used in Antonio Martins, Rio Grande do Norte, Brazil. Journal of Global Biosciences, v.4, n.8, p.3195-3200, 2015.

SILVA, T. P.; SILVA, E. M. J.; AMORIM, I. A.; AQUINO, A. L.; MATOS, T. E. S.; RODRIGUES, D. M. Levantamento de espécies vegetais e utilização em quintal agroflorestal de estabelecimento agrícola no assentamento Alegria - Marabá, Pará. Agroecossistemas, v.6, n. , p.103-109, 2014.

SIVIERO, A.; DELUNARDO, T. A.; HAVERROTH, M.; OLIVEIRA, L. C.; MENDONÇA, A. M. S. Plantas medicinais em quintais urbanos de Rio Branco, Acre. Revista Brasileira de Plantas Medicinais, Campinas, v.14, n.4, p.598$610,2011$.

STEPP, J. R.; MOERMAN, D. E. The importance of weeds in ethnopharmacology. Journal of Ethnopharmacology, v.75, p.19-23, 2001.

VALADÃO, L. M.; AMOROZO, M. C. M.; MOTTA, D. G. Produção de Alimentos na unidade domiciliar, dieta e estado nutricional: a contribuição dos quintais em um assentamento rural no estado de São Paulo. In: ALBUQUERQUE, U. P.; ALMEIDA, C. F. B. (Orgs.). Tópicos em Conservação e Etnobotânica de Plantas Alimentícias. Recife: Nuppea, 2006. p.92-115.

VIEIRA, T. A.; ROSA, L. S; SANTOS, M. M. L. S. Agrobiodiversidade de quintais agroflorestais no município de Bonito, Estado do Pará. Revista de Ciências Agrarias, Belém, v.55, n.3, p.159-166, 2012.

VISBISKI, V. N. Sistemas silvipastoris em Imaruí - SC: a construção de um sonho comum. 2003. 141f. Dissertação (Mestrado em Agrossistemas) - Centro de Ciências Agrárias. Universidade Federal de Santa Catarina, Florianópolis, 2003. 